\title{
Population Harvesting on both Prey and Predator
}

\author{
Aynalem Berhanie Emru, Purnachandra Rao Koya*, Mohammed Yiha Dawed
}

School of Mathematical and Statistical Sciences, Hawassa University, Hawassa, Ethiopia

Email address:

analemberhanie91@gmail.com (A. B. Emru),drkpraocecc@yahoo.co.in (P. R. Koya), mhmmdyiha@yahoo.com (M. Y. Dawed)

${ }^{*}$ Corresponding author

To cite this article:

Aynalem Berhanie Emru, Purnachandra Rao Koya, Mohammed Yiha Dawed. Population Harvesting on both Prey and Predator. American Journal of Applied Mathematics. Vol. 5, No. 3, 2017, pp. 91-98. doi: 10.11648/j.ajam.20170503.14

Received: April 29, 2017; Accepted: May 8, 2017; Published: June 29, 2017

\begin{abstract}
In this paper, the prey predator model as well as maximum sustainable yield has been discussed. Both prey and predator populations are considered to follow logistic law of growth. Both prey and predator harvesting or combined harvesting and maximum sustainable yield have been discussed. The maximum sustainable yield has been observed in two ways: as a function of one variable and also a function of two variables. Non-dimensionalization or scaling of the model, in order to reduce the number of the parameters has been performed. Positivity and boundedness of the solution have been studied. Stability analysis of the equilibrium point and also numerical simulations of the model in two dimensional as well as three dimensional cases have been done using MATLAB ode 45.
\end{abstract}

Keywords: Maximum Sustainable Yield, Stability, Combined Harvesting, Positivity and Boundedness, Simulation Study

\section{Introduction}

In an ecosystem, predation is a biological interaction where a predator feeds on its prey. Predator is a hunting organism while prey is the organism that is attacked. Predators may or may not kill their prey prior to feeding on them, but the act of predation often results in the death of the prey. However, predators are also often another organism's prey. For example, blue jays prey on insects, they in turn are prey for cats and snakes, and snakes are the prey for hawks.

Since most of predator-prey communities are linked it is interesting to study how they are linked to and affect each other. Studying of Predator-prey models plays an important role in the management of renewable resources.

A fishery is generally assumed to be a renewable resource because if a small number of fish from the population is harvested in a given year then those will be replaced by the by offspring of the adults during following year. Fishing has a lot of benefits to human beings. It serves as food, creates job opportunities and generates income. In general, it has great impact on the socioeconomic and infrastructure development of a country [1-3].

The optimal management of renewable resources is the maximum sustainable yield MSY of harvesting, being the maximum harvestable quantity. A population will go extinction over a period of time if it is harvested more than its MSY. That is, the population is over exploited. The MSY for the harvesting must be determined, in case the harvesting is allowed, for each population so as to assure the preservation of all species [4 - 7].

Many researchers modeled prey predator systems assuming that the predator will alive only if its prey exists. The generalist species predator-prey model including harvesting of both prey and predator has been studied. Here the predator is assumed to have alternative food resources [8-11].

The general practice is that the harvesting of prey and predator populations will be considered separately. However, in the present study harvesting of both prey and predator are considered. That is, the model can be used to study the combined harvesting of both the populations simultaneously. Harvesting is performed on both prey and predator populations at the same time.

The interaction of prey and predator is studied on the assumptions: (i) both the prey and predator populations grow following logistic functions (ii) there is high intra-specific competition among the predator population and (iii) harvesting is applied on both prey and predator populations, combined harvesting. 


\section{Construction of Mathematical Model}

The mathematical model for prey and predator can be constructed using Ordinary Differential Equations. Scaling of the model equations reduce the number of parameters helping to classify less and more important parameters. In the formulation of the model here the interesting issue is the maximum sustainable yield, also the harvesting of both populations conducted simultaneously.

The other issues of interest include performing of stability analysis of the equilibrium points, and numerical simulations. Simulation study of the model is conducted using MATLAB ode 45 software.

\subsection{Model Formulation}

In an existing prey-predator model constructed on the assumption that the prey and the predator population follow logistic law of growth, the population dynamics include the cases: (i) model without harvesting (ii) prey harvesting (iii) predator harvesting. In this study stability analysis and simulation study as well as boundedness and positivity of the model solution have been verified. However, the case of harvesting of both prey and predator simultaneously has not been considered [1].

To fill the gap, here the study is conducted on the maximum sustainable yield as well as species conservation considering the harvesting of both populations at the same time i.e., combined harvesting. The present model is built based on the following assumptions [1]:

(i) The variables $x(t)$ and $y(t)$ respectively represent the population sizes of prey and predator at time $t$.

(ii) The population sizes of both prey and predator follow logistic law of growth.

(iii) The predator consumes the prey population involving linearly interaction.

(iv) There is an intra-specific competition within the predator population.

(v) The harvesting rates of prey and predator populations are not the same.

(vi) Simultaneous harvesting of both prey and predator populations is possible.

The population dynamics of the system is then governed by the following system of differential equations:

$$
\begin{gathered}
d x / d t=r_{1} x\left[1-\left(x / k_{1}\right)\right]-a x y-q_{1} E x \\
d y / d t=r_{1} y\left[1-\left(y / k_{2}\right)\right]+b x y-\mu y^{2}-q_{2} E y
\end{gathered}
$$

Here in (1) - (2), $r_{1}$ and $r_{2}$ are respectively, the intrinsic growth rate of prey and predator populations; $k_{1}$ and $k_{2}$ are their respective environmental carrying capacities; $q_{1}$ and $q_{2}$ are the catchability coefficient of prey and predator population; $q_{1} E$ and $q_{2} E$ are the harvesting rates of prey and predator populations at time $t ; p$ is the predation rate; and $\mu$ is the coefficient of intra-specific competition among predators.

\subsection{Combined Harvesting}

In the combined harvesting, consider that the prey is
Tilapia fish with population size $x$ while the predator is Whale Fish with the population size $y$. The populations of both prey and predator are assumed to grow following logistic function. A variable $E$ is introduced into both the prey and predator equations and is called fishing effort. Assume that the catch of fish per unit effort is proportional to the availably amounts of $x$ and $y$. Thus, a population harvesting predator prey model with combined harvesting and intra-specific competition among predators is the same given by (1) and (2).

\section{Scaling the Combined Harvesting Model}

The scaled versions of the equations (1) - (2) using the new scaled parameters $u=x / k_{1}, v=y / k_{2}, t=$ $\left(1 / r_{1}\right) \tau$ take the form as

$$
\begin{gathered}
d u / d \tau=u(1-u)-a_{1} u v-E_{1} u \\
d v / d \tau=a_{2} v(1-v)+a_{3} u v-a_{4} v^{2}-E_{2} v
\end{gathered}
$$

Here in (3) - (4), $a_{1}=a k_{2} / r_{1}, a_{2}=r_{2} / r_{1}, a_{3}=$ $b k_{1} / r_{1}, \quad a_{4}=\mu k_{2} / r_{1}, \quad E_{1}=q_{1} E / r_{1}$ and $E_{2}=q_{2} E / r_{1}$.

\subsection{Boundedness of Solution of the Combined Harvesting Model}

Theorem: All solutions $\{u(\tau), v(\tau)\}$ of the system of model equations $(3)-(4)$ together with positive initial condition $\left(u_{0}, v_{0}\right)$ are bounded within the region $\Omega=$ $\left\{(u, v): 0 \leq u(\tau) \leq 1,0 \leq v(\tau) \leq\left[1+\left(a_{3} / a_{2}\right)\right]\right\}$.

Proof: The boundedness argument for $u(t)$ : the model equation (3) can be expressed as an inequality, without loss of generality, as $d u / d \tau \leq u(1-u)$. This inequality can be solved analytically to obtain a solution that sets an upper bound for $u(t)$. Using partial fraction method the inequality can be equivalently expressed as $\{(1 / u)+[1 /(1-u)]\} d u \leq d \tau$ and this can be solved to obtain a solution as $u(\tau) \leq\left[A /\left(A+e^{-\tau}\right)\right]$, where $A=$ $\left[u_{0} /\left(1-u_{0}\right)\right]$ and $u_{0}=u(0)$. Further, as $\tau \rightarrow \infty$ it can be observed that $u(\tau) \leq 1$. Thus, $\mathrm{u}(\tau)$ satisfies the boundary conditions that $u_{0} \leq u(\tau) \leq 1$ for all $\tau \geq 0$. Thus it can be concluded that $u(\tau)$ is bounded.

The boundedness argument for $v(t)$ : the model equation (4) can be expressed as an inequality, without loss of generality, as $d v / d \tau \leq a_{2} v(1-v)+a_{3} v$. This inequality can be solved analytically to obtain a solution that sets an upper bound for $v(t)$. Using partial fraction method the inequality can be equivalently expressed as $\left[1 /\left(a_{2}+a_{3}\right)\right]\left\{(1 / v)+a_{2}\left[a_{2}(1-v)+a_{3}\right]\right\} \leq d \tau$ and this can be solved to obtain a solution as $v(\tau) \leq\left\{B\left(a_{2}+a_{3}\right) /\left[a_{2} B+e^{-\left(a_{2}+a_{3}\right) \tau}\right]\right\}$, where $B=\left\{v_{0} /\left[a_{2}\left(1-v_{0}\right)+a_{3}\right]\right\}$ and $v_{0}=v(0)$. Further, as $\tau \rightarrow \infty$ it can be observed that $v(\tau) \leq\left[1+\left(a_{3} / a_{2}\right)\right]$. Thus, $v(\tau)$ satisfies the boundary conditions that $v_{0} \leq v(\tau) \leq$ $\left[1+\left(a_{3} / a_{2}\right)\right]$ for all $\tau \geq 0$. Thus it can be concluded that $v(\tau)$ is bounded.

Therefore all solutions of the system of model equations (3) - (4) with positive initial values in $R_{2}{ }^{+}$are bounded in the region $\Omega$. 


\subsection{Positivity of the Solution}

In the present model population sizes of the prey as well as the predator should not be negative because population cannot be negative. Hence the solutions of (1) - (2) are expected to be positive quantities. It is sufficient to show that the solution of the scaled model expressed in (3) - (4) is nonnegative and the same is done as follows:

Non - negativity of $v(\tau)$ : the equation (4), without loss of generality, can be expressed as $d v / d \tau \geq-v\left(a_{2} v+a_{4} v+\right.$ $\left.E_{2}\right)$. Using integration by part it is possible to find its general solution. After simplification the solution takes the form as $v(\tau)=\left\{A E_{2} /\left[e^{E_{2} \tau}-A\left(a_{2}+a_{4}\right)\right]\right\}$, here $A=$ $v_{0} /\left[E_{2}+\left(a_{2}+a_{4}\right) v_{0}\right]$ is a constant, and $v_{0}=v(0)$ is the initial population size of $v(\tau)$. Further it can be observed that $\lim v(\tau) \rightarrow 0$ as the time $\tau \rightarrow \infty$. Hence, the variable $v(\tau)$ is non - negative for all $\tau \geq 0$.

Non - negativity of $u(\tau)$ : The equation (3), without loss of generality, can be expressed as $d u / d \tau \geq-u^{2}-a_{1} u v-$ $E_{1} u$. Since minimum of $v$ is zero, the foregoing inequality can be equivalently put as $d u / d \tau \geq-u^{2}-E_{1} u$. Using integration by part it is possible to find its general solution. After simplification the solution takes the form as $u(\tau) \geq$ $\left\{E_{1} /\left[B e^{E_{1} \tau}-1\right]\right\}$, here $B=1-\left(E_{1} / u_{0}\right)$ is a constant, and $u_{0}=u(0)$ is the initial population size of $u(\tau)$. Further it can be observed that $\lim u(\tau) \rightarrow 0$ as the time $\tau \rightarrow \infty$. Hence, the variable $u(\tau)$ is non - negative for all $\tau \geq 0$.

Therefore both $v(\tau)$ and $u(\tau)$ are non - negative quantities for all the values of temporal coordinate $\tau$.

\subsection{Equilibrium Points}

From the scaled equations (3) - (4) of the model, the equilibrium points can be found by setting the left hand sides equal to zero and solving the resultant equations. Thus the equilibrium points $(u, v)$ are solutions of the pair of equations $u(1-u)-a_{1} u v-E_{1} u=0$ and $a_{2} v(1-v)+$ $a_{3} u v-a_{4} v^{2}-E_{2} v=0$. In all there are four equilibrium points: one is trivial; two are axial; and one is interior as illustrated below:

(i) The trivial equilibrium point is $(0,0)$. It occurs at the origin.

(ii) The axial equilibrium points are $\left\{0,\left[a_{2}-E_{2} / a_{2}+a_{4}\right]\right\}$ and $\left(1-E_{1}, 0\right)$. The former is on $v$ axis while the other occurs on $u$ axis.

(iii) The interior equilibrium point occurs in the positive quadrant of $u v$ plane at

$$
\left(u^{*}, v^{*}\right)=\left\{\frac{\left(a_{2}+a_{4}\right)\left(1-E_{1}\right)+a_{1}\left(E_{2}-a_{2}\right)}{a_{2}+a_{1} a_{3}+a_{4}}, \frac{a_{2}+a_{3}-\left(a_{3} E_{1}+E_{2}\right)}{a_{2}+a_{1} a_{3}+a_{4}}\right\}
$$

Note that the axial equilibrium points are valid only if the parameters satisfy $E_{2}<a_{2}$ and $E_{1}<1$. In addition to these the interior equilibrium point is valid if and only if $\left\{a_{2}-\right.$ $\left.\left[\left(a_{2}+a_{4}\right)\left(1-E_{1}\right) / a_{1}\right]\right\}<E_{2}$. Thus, it can be concluded that all the four equilibrium points are valid if the following conditions of parameters are valid:

$$
\begin{gathered}
0<E_{1}<1 \\
\left\{a_{2}-\left[\left(a_{2}+a_{4}\right)\left(1-E_{1}\right) / a_{1}\right]\right\}<E_{2}<a_{2}
\end{gathered}
$$

\subsection{Stability Analysis of the Equilibrium Points}

In order to verify stability of the equilibrium point, the first and foremost thing to be done is finding the Jacobin matrix $J(u, v)$ of the functions $f_{1}(u, v)=u(1-u)-a_{1} u v-E_{1} u$ and $f_{2}(u, v)=a_{2} v(1-v)+a_{3} u v-a_{4} v^{2}-E_{2} v$. The element form of Jacobin matrix can be expressed as

$$
J(u, v)=\left[\begin{array}{ll}
\frac{\partial f_{1}}{\partial u} & \frac{\partial f_{1}}{\partial v} \\
\frac{\partial f_{2}}{\partial u} & \frac{\partial f_{2}}{\partial v}
\end{array}\right]=\left[\begin{array}{cc}
1-2 u-a_{1} v-E_{1} & a_{2} u \\
a_{3} v & a_{2}-2 a_{2} v+a_{3} u-2 a_{4} v-E_{2}
\end{array}\right]
$$

Theorem 1: The trivial equilibrium point $(0,0)$ of the model (3) - (4) is unstable.

Proof: Note that an equilibrium point is said to be unstable if the eigenvalues of the Jacobian matrix at that point are positive. Now the Jacobian matrix (8) at the trivial equilibrium point $(0,0)$ reduces to the form as

$$
J(u, v)=\left[\begin{array}{cc}
1-E_{1} & 0 \\
0 & a_{2}-E_{2}
\end{array}\right]
$$

The characteristic equation $|J(0,0)-\lambda I|=0$ is thus found to be $\left(1-E_{1}-\lambda\right)\left(a_{2}-E_{2}-\lambda\right)=0$ giving two eigenvalues as $\lambda_{1}=1-E_{1}$ and $\lambda_{2}=a_{2}-E_{2}$. From the restrictions (6) and (7) set on parameters it can be verified that both the eigenvalues $\lambda_{1}$ and $\lambda_{2}$ are positive. Therefore the trivial equilibrium point $(0,0)$ is unstable.

Theorem 2: The axial equilibrium point $\left(1-E_{1}, 0\right)$ of the model (3) - (4) is unstable.

Proof: Note that an equilibrium point is said to be unstable if among the two eigenvalues of the Jacobian matrix at that point, one is positive and the other is negative.
Now the Jacobian matrix (8) at the axial equilibrium point $\left(1-E_{1}, 0\right)$ reduces to the form as

$$
J\left(1-E_{1}, 0\right)=\left[\begin{array}{cc}
E_{1}-1 & -a_{1}\left(1-E_{1}\right) \\
0 & a_{2}+a_{3}\left(1-E_{1}\right)-E_{2}
\end{array}\right]
$$

The characteristic equation $|J-\lambda I|=0$ is thus found to be $\left(E_{1}-1-\lambda\right)\left(a_{2}+a_{3}\left(1-E_{1}\right)-E_{2}\right)=0$ giving two eigenvalues as $\lambda_{1}=E_{1}-1$ and $\lambda_{2}=a_{2}+a_{3}\left(1-E_{1}\right)-E_{2}$. From the restrictions (6) and (7) set on parameters it can be verified that $\lambda_{1}$ is negative while $\lambda_{2}$ is positive. Therefore the axial equilibrium point $\left(1-E_{1}, 0\right)$ is unstable.

Theorem 3: The axial equilibrium point $\left\{0,\left[\left(a_{2}+E_{2}\right) /\left(a_{2}+a_{4}\right)\right]\right\}$ of the model (3) - (4) is unstable.

Proof: Note that an equilibrium point is said to be unstable if among the two eigenvalues of the Jacobian matrix at that point, one is positive and the other is negative.

Now the Jacobian matrix (8) at the axial equilibrium point $\left\{0,\left[\left(a_{2}-E_{2}\right) /\left(a_{2}+a_{4}\right)\right]\right\}$ reduces to the form as 


$$
J=\left[\begin{array}{cc}
1-E_{1}\left(\frac{a_{2}-E_{2}}{a_{2}+a_{4}}\right) & 0 \\
a_{3}\left(\frac{a_{2}-E_{2}}{a_{2}+a_{4}}\right) & E_{2}-a_{2}
\end{array}\right]
$$

The characteristic equation $|J-\lambda I|=0$ is thus computed to be $\left\{1-E_{1}-\left[\left(a_{2}-E_{2}\right) /\left(a_{2}+a_{4}\right)\right]-\lambda\right\}=0$ giving two eigenvalues as $\quad \lambda_{1}=E_{2}-a_{2}$ and $\lambda_{2}=\left\{\left[\left(a_{2}+a_{4}\right)\left(1-E_{1}\right)-a_{1}\left(a_{2}-E_{2}\right)\right] /\left(a_{2}+a_{4}\right)\right\}$. From the restrictions (6) and (7) set on parameters it can be verified that $\lambda_{1}$ is negative while $\lambda_{2}$ is positive. Therefore the axial equilibrium point $\left\{0,\left[\left(a_{2}-E_{2}\right) /\left(a_{2}+a_{4}\right)\right]\right\}$ is unstable.

Furthermore, the stability of the equilibrium point can be done making the use of Jacobian matrix. Let $\left(u^{*}, v^{*}\right)$ be the positive equilibrium point. The determinant and the trace of the Jacobian at $\left(u^{*}, v^{*}\right)$ at is given by $\operatorname{det}(J)=a_{2}+a_{3} u^{*}+$ $E_{2}\left(2 u^{*}+E_{1}-1\right)+\left(a_{2}+a_{4}\right)\left(4 u^{*} v^{*}+2 a_{1} v^{*}+2 E_{1} v^{*}\right)-$ $\left(\left(a_{2}+a_{4}\right)\left(2 v^{*}\right)+\left(2 u^{*}+E_{1}\right)\left(a_{2}+a_{3} u^{*}\right)\right)$ and $\operatorname{tr}(J)=$ $1-E_{1}+a_{2}-E_{2}+a_{3} u^{*}-\left(2 u^{*}+\left(a_{1}+2 a_{2}+2 a_{4}\right) v^{*}\right)$. Here it is required that the $\operatorname{determinant} \operatorname{det}(J)$ is positive while the trace $\operatorname{tr}(J)$ is negative. Hence, this requirement leads to the following restrictions on the parameters:

$$
\begin{array}{r}
1-E_{1}+a_{2}-E_{2}+a_{3} u^{*}<2 u^{*}+\left(a_{1}+2 a_{2}+2 a_{4}\right) v^{*} \\
{\left[a_{2}+a_{3} u^{*}+E_{2}\left(2 u^{*}+E_{1}-1\right)+\left(a_{2}+a_{4}\right)\left(4 u^{*} v^{*}+\right.\right.} \\
\left.\left.2 a_{1} v^{*}+2 E_{1} v^{*}\right)\right]>\left[\left(a_{2}+a_{4}\right)\left(2 v^{*}\right)+\left(2 u^{*}+E_{1}\right)\left(a_{2}+\right.\right. \\
\left.\left.a_{3} u^{*}\right)\right]
\end{array}
$$

Theorem 4: The interior equilibrium point $\left(u^{*}, v^{*}\right)$ is stable if the two conditions (9) and (10) are satisfied:

Proof: If condition (9) is satisfied then the trace is less than zero and if condition (10) is satisfied determinant is greater than zero. Therefore, the interior equilibrium point $\left(u^{*}, v^{*}\right)$ is stable if the above two conditions are satisfied.

\subsection{Maximum Sustainable Yield}

A sustainable yield is an amount of fish in the water that is required to keep on breeding so that new generations of fish are created. The maximum sustainable yield or MSY is the largest amount of fish that can be taken out of the water, yet leaving enough to ensure sustainability i.e., to ensure there are enough fish in the water to produce new generations. Maximum sustainable yield of any stock is the greatest yield that can be achieved over time while maintaining productive capacity of the stock [11].

A number of factors contribute to determine MSY for any species. These include how fast they grow, when and how they reproduce and the pattern of harvesting in the fishery. Typically MSY for a fish stock is also variable over time, because of changes in productivity and environmental factors. Recall that for the scaled equations (3) and (4) the interior equilibrium point $\left(u^{*}, v^{*}\right)$ is given by (5).
The sustainable yield during the equilibrium state is denoted and can be expressed following the procedure given in [2]. Thus, the yield in the equilibrium situation is defined as

$$
Y\left(E_{1}, E_{2}\right)=Y\left(E_{1}\right)+Y\left(E_{2}\right)=E_{1} u^{*}+E_{2} v^{*}
$$

Here the main objective is to find the maximum sustainable yield i.e., to find the point $\left(E_{1}, E_{2}\right)$ at which the function $Y\left(E_{1}, E_{2}\right)$ defined in (11) gains a maximum value.

In case of a function of one variable, the local maximum or minimum is found using differentials of the given function. The local maximum or minimum occurs where the first derivative of the function equals to zero. The point at which the first derivative of the function is zero and the second derivative is negative is called maximum point. Further, the point at which the first derivative of the function is zero and second derivative is positive is called minimum point.

\subsection{Finding Stationary Points of a Function of Two Variables}

In the present case the yield function is a function $Y\left(E_{1}, E_{2}\right)$ of two variables $E_{1}$ and $E_{2}$. So in order to find the maximum yield point the following procedure can be followed: A point $\left(c^{*}, d^{*}\right)$ which is either maximum or minimum or saddle point is commonly known as a stationary point. The computed value of a function at the stationary point is called the stationary value of that function. To find the stationary points of $Y\left(E_{1}, E_{2}\right)$ the partial derivatives $\partial Y / \partial E_{1}$ and $\partial Y / \partial E_{1}$ are required to be worked out and set both equal to zero. This set up gives two equations in two unknowns $E_{1}$ and $E_{2}$. Solving these equations will provide maximum yielding population sizes of prey and predator $E_{1}$ and $E_{2}$. In what follows the concept of classifying stationary points is briefly summarized.

The classification of a stationary point of a function of two variables is provided here. The general notations for representing partial derivatives include $\quad Y_{E_{1}}=\partial Y / \partial E_{1}, Y_{E_{2}}=\partial Y / \partial E_{2}, Y_{E_{1} E_{1}}=$ $\partial^{2} Y / \partial E_{1}^{2}, Y_{E_{2} E_{2}}=\partial^{2} Y / \partial E_{2}^{2}, Y_{E_{1} E_{2}}=Y_{E_{2} E_{1}}=$

$\partial^{2} Y / \partial E_{1} \partial E_{2}=\partial^{2} Y / \partial E_{2} \partial E_{1}$. Recall that at a stationary point $\left(u^{*}, v^{*}\right)$ the function $Y\left(E_{1}, E_{2}\right)$ satisfies the conditions $Y_{E_{1}}=0$ and $Y_{E_{2}}=0$. Further, (i) if $\left[Y_{E_{1} E_{1}} Y_{E_{2} E_{2}}-\right.$ $\left.\left(\partial^{2} Y / \partial E_{1} \partial E_{2}\right)\right]<0$ then $\left(c^{*}, d^{*}\right)$ is a saddle point (ii) if $\left[Y_{E_{1} E_{1}} Y_{E_{2} E_{2}}-\left(\partial^{2} Y / \partial E_{1} \partial E_{2}\right)\right]>0$ then $\left(c^{*}, d^{*}\right)$ is either maximum or minimum point (iii) In addition to the satisfaction of the condition given in (ii) if $Y_{E_{1} E_{1}}<0$ and $Y_{E_{2} E_{2}}<0$ then $\left(c^{*}, d^{*}\right)$ is the maximum point and finally (iv) in addition to the satisfaction of the condition given in (ii) if $Y_{E_{1} E_{1}}>0$ and $Y_{E_{2} E_{2}}>0$ then $\left(c^{*}, d^{*}\right)$ is the minimum point.

The explicit expression the yield function (11) and its first and second order partial derivatives can be expressed as

$$
Y\left(E_{1}, E_{2}\right)=\frac{\left(a_{2}+a_{4}\right)\left(E_{1}-E_{2}^{2}\right)+\left(a_{1}-a_{3}\right) E_{1} E_{2}+E_{2}\left(a_{2}+a_{3}-E_{2}\right)-E_{1} a_{1} a_{2}}{a_{2}+a_{1} a_{3}+a_{4}}
$$




$$
\begin{gathered}
\frac{\partial Y}{\partial E_{1}}=\frac{a_{2}+a_{4}-2 a_{2} E_{1}-2 a_{4} E_{1}-a_{1} a_{2}+E_{2} a_{1}-a_{3} E_{2}}{a_{2}+a_{1} a_{3}+a_{4}} \\
\frac{\partial Y}{\partial E_{2}}=\frac{E_{1} a_{1}+a_{2}+a_{3}-a_{3} E_{1}-2 E_{2}}{a_{2}+a_{1} a_{3}+a_{4}} \\
\frac{\partial^{2} Y}{\partial E_{1}{ }^{2}}=\frac{-2 a_{2}-2 a_{4}}{a_{2}+a_{1} a_{3}+a_{4}} \\
\frac{\partial^{2} Y}{\partial E_{2}{ }^{2}}=\frac{-2}{a_{2}+a_{1} a_{3}+a_{4}} \\
\frac{\partial^{2} Y}{\partial E_{1} E_{2}}=\frac{\partial^{2} Y}{\partial E_{2} E_{1}}=\frac{\left(a_{1}-a_{3}\right)}{a_{2}+a_{1} a_{3}+a_{4}}
\end{gathered}
$$

In view of the above solving the two equations $\partial Y / \partial E_{1}=$ $\partial Y / \partial E_{2}=0$ leads to one stationary point $\left(c^{*}, d^{*}\right)$ where

$$
\begin{gathered}
c^{*}=\left[\frac{\left(2 a_{1}+8 a_{2}-2 a_{3}\right)\left(a_{2}+a_{4}\right)+\left(a_{3}-a_{2}\right)\left(a_{1}{ }^{2}+a_{3}{ }^{2}\right)-2 a_{1} a_{3}{ }^{2}}{\left(a_{1}-a_{3}\right)^{2}\left(a_{3}-a_{1}\right)+4\left(a_{1}-a_{3}\right)\left(a_{3}+a_{2}\right)}\right] \\
d^{*}=\left[\frac{\left(a_{1}+a_{3}+2 a_{2}\right)\left(a_{2}+a_{4}\right)-a_{1} a_{2}\left(a_{1}-a_{3}\right)}{\left(a_{3}-a_{1}\right)\left(a_{1}-a_{3}\right)+4\left(a_{2}+a_{3}\right)}\right]
\end{gathered}
$$

It is now need to classify $\left(c^{*}, d^{*}\right)$. Now the determinant is given by

$$
\frac{\partial^{2} Y}{\partial E_{1}} \frac{\partial^{2} Y}{\partial E_{2}{ }^{2}}-\frac{\partial^{2} Y}{\partial E_{1} E_{2}}=\frac{2\left(2 a_{2}+2 a_{4}+a_{1} a_{3}\right)-\left(a_{1}+a_{3}\right)^{2}}{\left(a_{2}+a_{1} a_{3}+a_{4}\right)}
$$

Here it can be observed that the determinant given in (14) is greater than zero if the condition on parameters $2\left(2 a_{2}+\right.$ $\left.2 a_{4}+a_{1} a_{3}\right)>\left(a_{1}+a_{3}\right)^{2}$ holds true. However, the conditions $\partial^{2} Y / \partial E_{1}{ }^{2}<0$ and $\partial^{2} Y / \partial E_{2}{ }^{2}<0$ do hold absolutely true. It can be concluded that the maximum sustainable yield $Y_{M S Y}=E_{1} c^{*}+E_{2} d^{*}$ is given at the point $\left(c^{*}, d^{*}\right)$ where the expressions for $c^{*}$ and $d^{*}$ are given by (12) and (13) respectively provided that the following condition on parameters hold

$$
2\left(2 a_{2}+2 a_{4}+a_{1} a_{3}\right)>\left(a_{1}+a_{3}\right)^{2}
$$

Table 1. Model parameters and their description.

\begin{tabular}{ll}
\hline Parameter & Description \\
\hline$r_{1}$ & The intrinsic growth rate of prey population \\
$r_{2}$ & The intrinsic growth rate of predator population \\
$q_{1}$ & The catchability coefficient of prey population \\
$q_{2}$ & The catchability coefficient of predator population \\
$q_{1} E$ & The harvesting rate from prey population at time $\mathrm{t}$ \\
$q_{1} E$ & The harvesting rate from predator population at time t \\
$a$ and $b$ & The predation rates \\
$\mu$ & The coefficient of intra-specific competition among \\
\hline
\end{tabular}

\section{Numerical Simulations}

Numerical simulations of the model have been carried out using MATLAB inbuilt function ode 45 . The simulation study is carried out in two ways for the maximum sustainable yield: such as the maximum sustainable yield as a function of one variable and maximum sustainable yield as function of two variables have been studied. The graph for a function of one variable as well as function of two variables has been discussed through 2 and 3 dimensional plot as follows:

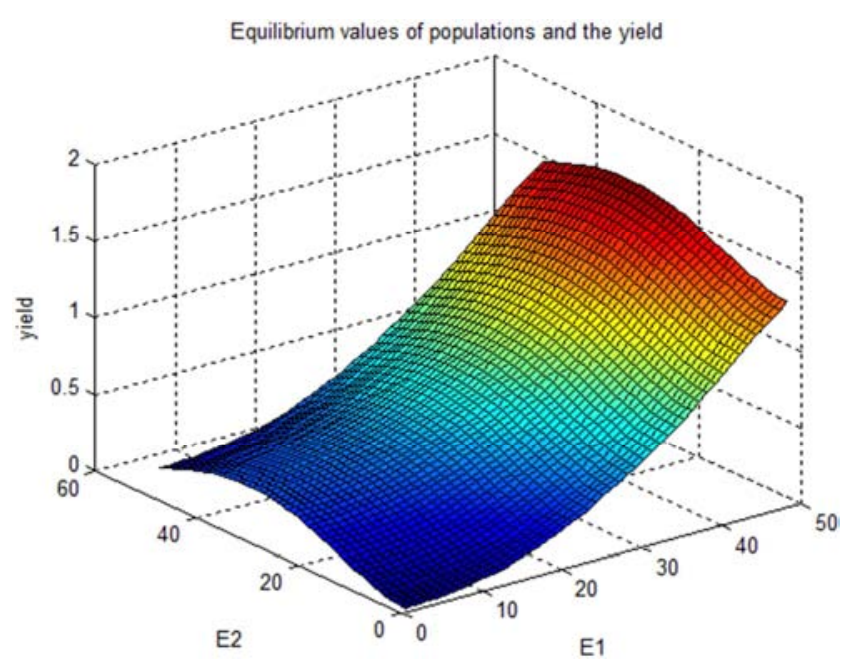

Figure 1. Dynamics of population biomass with $a_{1}=0.999, a_{2}=0.8, a_{3}=$ $0.99, a_{4}=0.71, E_{1}=0.8, E_{2}=0.6, \beta=0.28, a_{5}=0.5, u_{0}=5, v_{0}=3$.

In Figure 1, the simulation study shows that the maximum sustainable yield of the combined population is around $Y_{M S Y}=1.642$ units. This combined yield occurs when the efforts of prey and predator are $\left(E_{1}, E_{2}\right)=(49,33)$. Further, it can be observed that the maximum yield increasing $E_{2}$ increases from 0 to 40 and decreases thereafter.

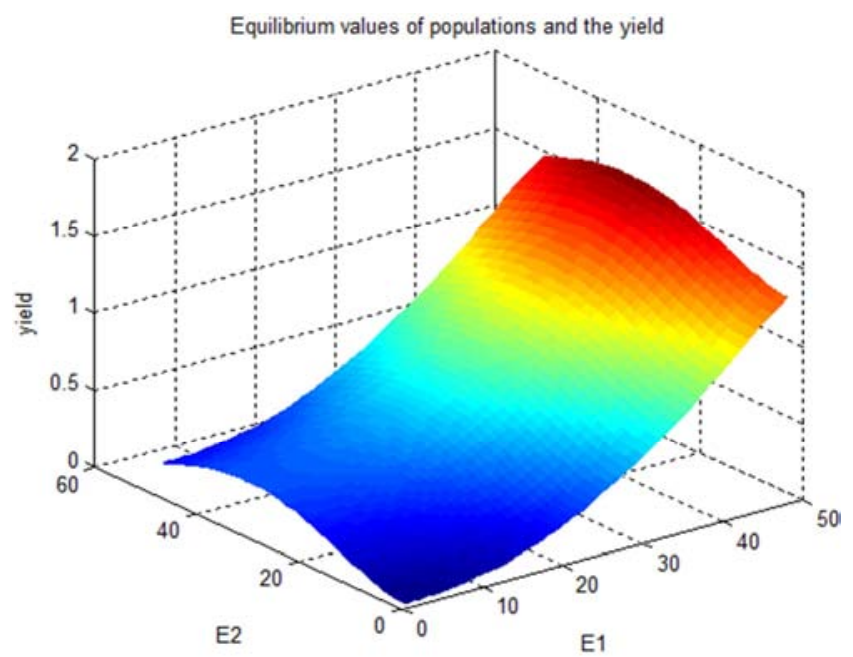

Figure 2. Dynamics of population biomass with $a_{1}=0.999, a_{2}=0.8, a_{3}=$ $0.99, a_{4}=0.71, E_{1}=0.48, E_{2}=0.6, \beta=0.28, a_{5}=0.5, u_{0}=5, v_{0}=3$.

In Figure 2, the simulation study shows that the maximum sustainable yield of the combined population is around $Y_{M S Y}=1.643$ units. This combined yield occurs when the efforts of prey and predator are $\left(E_{1}, E_{2}\right)=$ $(49,32)$. Further, it can be observed that the maximum yield increasing $E_{2}$ increases from 0 to 52 and decreases thereafter. 


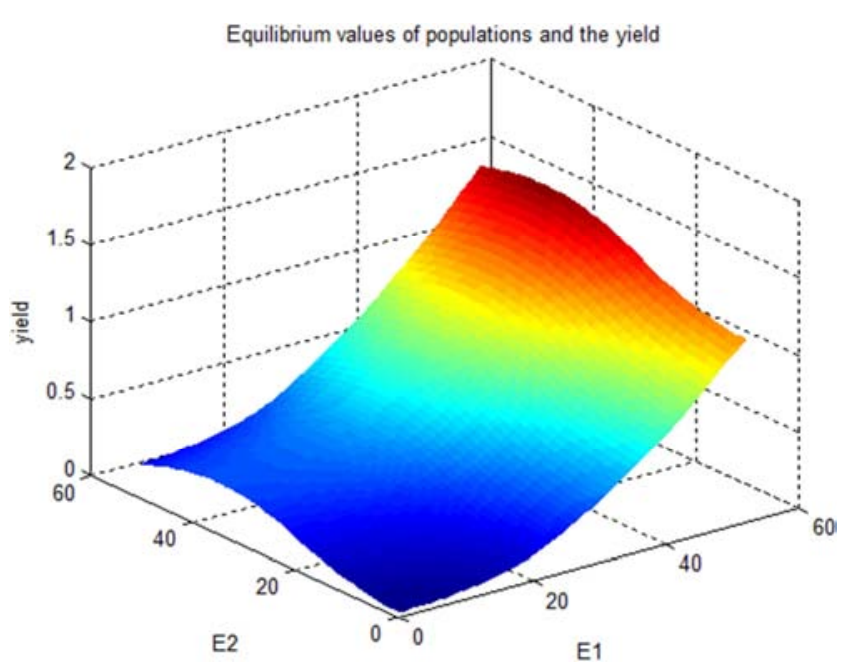

Figure 3. Dynamics of population biomass with $a_{1}=0.99, a_{2}=0.99, a_{3}=$ $0.79, a_{4}=0.88, E_{1}=0.28, E_{2}=0.1, \beta=0.118, a_{5}=0.5, u_{0}=9, v_{0}=5$.

In Figure 3, the simulation study shows that the maximum sustainable yield of the combined population is around $Y_{M S Y}=1.575$ units. This combined yield occurs when the efforts of prey and predator are $\left(E_{1}, E_{2}\right)=(53,40)$. Further, it can be observed that the maximum yield increasing $E_{2}$ increases from 0 to 40 and decreases thereafter.

Alternative way of finding Maximum sustainable yield in 2D: The maximum sustainable yield for combined harvesting can be also found in two dimensional i.e., function of one variable by rescaling the model. This technique will be applied on the model equations (1) - (2) by considering new scaling parameters as $u=x / k_{1}, v=y / k_{2}, t=\left(1 / r_{1}\right) \tau$. In terms of these new scaling parameters the equations take the form as:

$$
\begin{gathered}
d u / d \tau=u(1-u)-a_{1} u v-\beta E u \\
d v / d \tau=a_{2} v(1-v)+a_{3} u v-a_{4} v^{2}-a_{5} E v
\end{gathered}
$$

Here in (14) - (15), the notations used and their expressions include $a_{1}=a k_{2} / r_{1}, a_{2}=r_{2} / r_{1}, a_{3}=$ $a k_{1} / r_{1}, \quad a_{4}=\mu k_{2} / r_{1}, \quad \beta=q_{1} / r_{1}, \quad a_{5}=q_{2} / r_{1}$.

On setting the left hand sides system of two equations (16) - (17) to zero and after making some algebraic simplifications the interior or co-existence equilibrium point can be obtained as

$\left(u^{* *}, v^{* *}\right)$

$=\left(\frac{\left(a_{2}+a_{4)(1-\beta E)}+a_{1}\left(a_{5} E-a_{2}\right)\right.}{\left(a_{2}+a_{1} a_{3}+a_{4}\right)}, \frac{a_{2}+a_{3}-\left(a_{3} \beta E+a_{5} E\right)}{\left(a_{2}+a_{1} a_{3}+a_{4}\right)}\right)$

As stated in [2] the yield function in the equilibrium situation is defined as $Y(E)=E u^{* *}+E v^{* *}$ and hence it takes the expression as

$$
Y(E)=E\left[\frac{\left(a_{2}+a_{3}+a_{4}\right)(1-\beta E)+\left(a_{2}-a_{5} E\right)\left(1-a_{1}\right)}{\left(a_{2}+a_{1} a_{3}+a_{4}\right)}\right]
$$

To find the maximum sustainable total yield MSTY the first derivative of the yield function $Y(E)$ with respect to effort $E$ is to be constructed and made equal to zero. Then solve for the stationary point. It is a point at which the first derivative of a function equal to zero. Inserting of the stationary point in to the yield function $Y(E)$ results the maximum sustainable yield. To find the MSTY a procedure is followed as follows:

$$
\frac{d Y}{d E}=\left[\frac{\left(a_{2}+a_{3}+a_{4}\right)(1-2 \beta E)+\left(a_{2}-2 a_{5} E\right)\left(1-a_{1}\right)}{\left(a_{2}+a_{1} a_{3}+a_{4}\right)}\right]
$$
$E=$

Setting $d Y / d E=0$ and solving gives the result as $\left\{\left(2 a_{2}+a_{3}+a_{4}-a_{1} a_{2}\right) /\left[2\left(a_{2} \beta+a_{3} \beta+a_{4} \beta+a_{5}-a_{1} a_{5}\right)\right]\right\}$ provided that the denominator is not zero, that is $a_{2} \beta+$ $a_{3} \beta+a_{4} \beta+a_{5} \neq a_{1} a_{5}$. Hence the MSTY is given be

$$
Y_{M S T Y}=\left(u^{* *}+v^{* *}\right)\left[\frac{2 a_{2}+a_{3}+a_{4}-a_{1} a_{2}}{2\left(a_{2} \beta+a_{3} \beta+a_{5}-a_{1} a_{5}\right)}\right]
$$

A simulation study is conducted on the model equations (14) - (15) and on the yield function (16). The results are presented in the form of figures below:
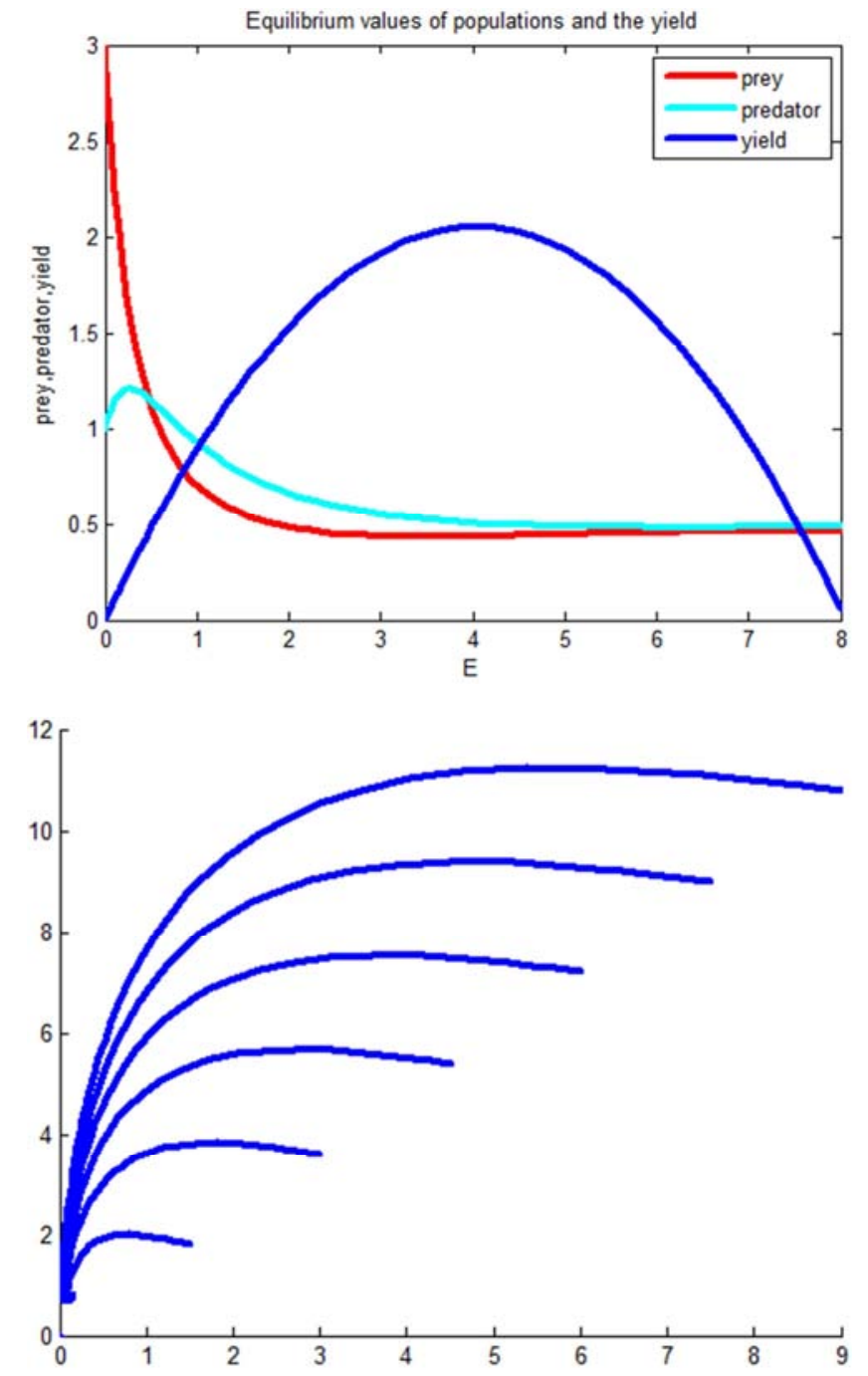

Figure 4. Time series plot and phase diagram of population biomass for the parametric values $a_{1}=0.97, a_{2}=0.53, a_{3}=0.99, a_{4}=0.99, E=$ $0.5, \beta=0.1189, a_{5}=0.5,\left(u_{0}, v_{0}\right)=(3,1)$.

In Figure 4, the time series plot of the system (14) - (16) 
indicates that population sizes of both prey and predator converge to a finite value. The phase portrait shows that the interior equilibrium point $(0.46689,0.4883)$ is stable. The yield curve is increasing for any effort lying in the interval $E \in(0,4.046)$ and decreases thereafter. The maximum sustainable yield is 2.052 at $E=4.046$. As it can be observed the prey biomass decreases when effort $0 \leq E \leq 3.246$ and increases when the effort is $3.246 \leq E \leq 10$. The predator biomass increases when $0 \leq E \leq 0.24$ and decreases when $0.24 \leq E \leq 10$.
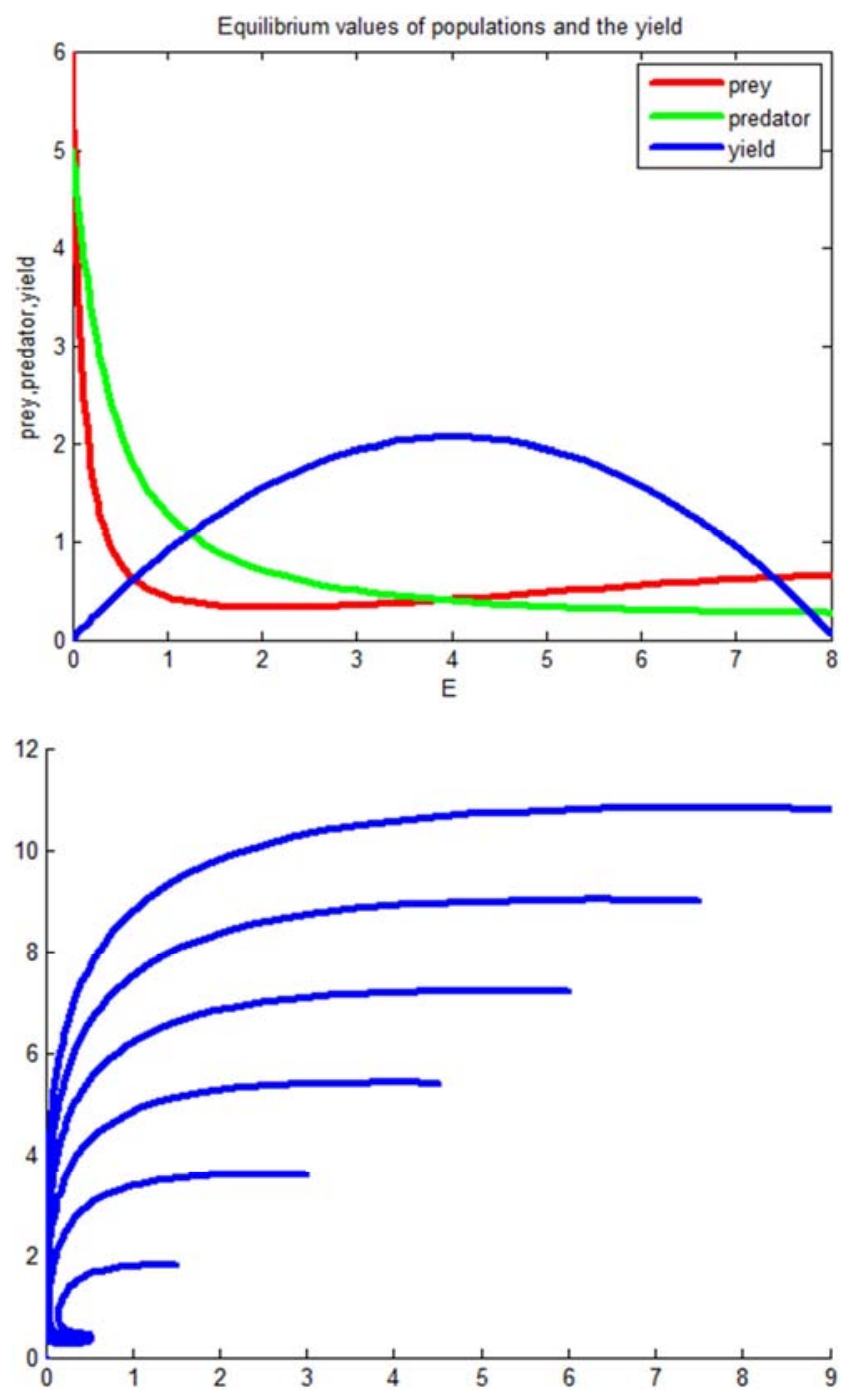

Figure 5. Time series plot and phase diagram of population biomass for the parametric values $a_{1}=0.95, a_{2}=0.2, a_{3}=0.3, a_{4}=0.49, E=$ $0.5, \beta=0.1189, a_{5}=0.5$, and the initial population $\left(u_{0}, v_{0}\right)=(6,5)$.

In Figure 5, the time series plot of the system (13) - (14) indicates that population size converges to finite equilibrium value. The phase portrait of the system in this figure corresponding to different initial values indicates that the interior equilibrium point $(0.7143,0.2381)$ is stable. The yield curve is increasing for any effort lying in $(0,4.046)$. The maximum sustainable yield is 2.068 at $E=4.068$. It is observed in figure 5 the prey biomass decreases when effort $0 \leq E \leq 1.5$, constant when effort $1.5 \leq E \leq 2.8$ and increases when effort $2.8 \leq E \leq 8$. While the predator biomass decreases throughout the domain $\mathrm{E}$, but at the beginning it decreases rapidly after a while the change is very slow.
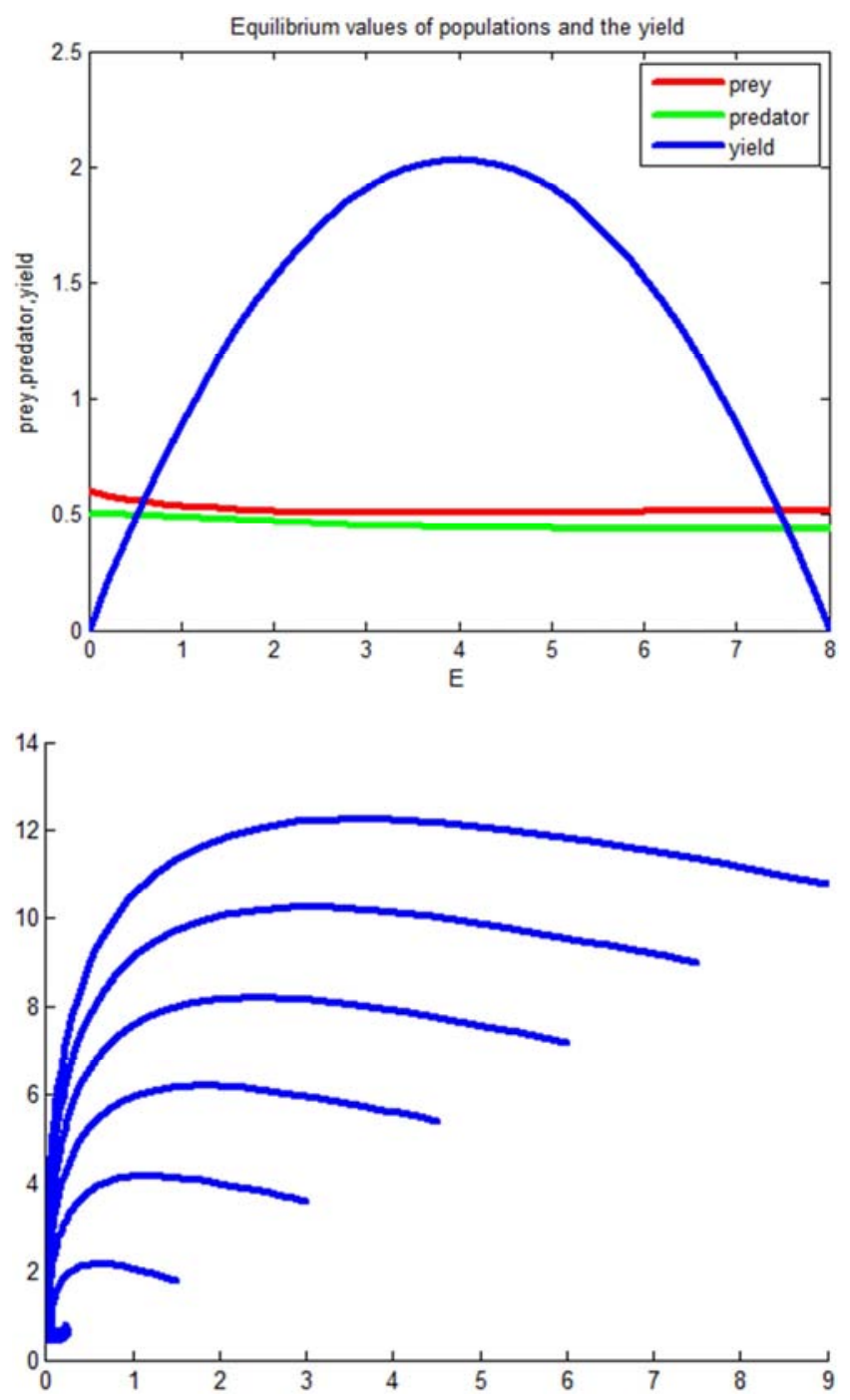

Figure 6. Time series plot and phase diagram of population biomass for the parametric values $a_{1}=0.97, a_{2}=0.3, a_{3}=0.99999, a_{4}=0.99, E=$ $0.5, \beta=0.1189, a_{5}=0.5$ and the initial population $\left(u_{0}, v_{0}\right)=$ $(0.6,0.5)$

In Figure 6, the phase portrait of the system corresponding to differential values indicates that the interior equilibrium point $(0.5154,0.43829)$ is stable. The yield curve is increasing for any effort lying in $(0,4.01)$. The maximum sustainable yield is 2.035 at $E=4.01$. It can be observed that biomass of the prey as well as the predator changes almost constant throughout effort.

\section{Conclusions}

In this paper, a modified mathematical model is proposed to describe the maximum combined harvesting on prey and predator population as well as maximum sustainable yield. The stability analysis and numerical simulations for the maximum sustainable yield have been performed. From the stability analysis of the equilibrium point it could be stable 
only under some specific conditions. The numerical simulations for the maximum sustainable yield have been seen in two different ways. That is yield as a function of one variable and function of two variables have been also done. Positivity and boundedness of the model also have been studied. Since, the numerical simulation studies and stability analysis of the model are conducted the model could be applied to the real-world situations, and may help to get maximum sustainable harvest without extremely affecting the fish population in the environment and also to keep the preypredator relationship in balanced condition.

\section{References}

[1] Mohammed Yiha Dawed, Purnachandra Rao Koya and Temesgen Tibebu Mekonen, Generalist species predator-Prey model and maximum sustainable yield, IOSR Journal of Mathematics (IOSR-JM), Vol. 12, Issue 6, Ver. V, 2016, Pp 13-24. DOI: $10.9790 / 5728-1206051324$

[2] Susmita Paul, Paritosh Bhattacharya, K. S. Choudhury, Maximum Sustainable Yield Policy in Prey-Predator System-a study, 2013.
[3] Kinfe Hailemariam Hntsa, Zenebe Taka Mengesha, Mathematical modeling of Fish Resources Harvesting with Predator at Maximum Sustainable Yield, 15 June 2016.

[4] Jicai Huang and Yijun Gong, Bifurcation analysis in preypredator model with constant-yield predator harvesting.

[5] Mohamed Faris Laham, Ishtrinayagy S. Krishnarajah and Jamilah Mohd Shariff, Fish Harvesting Management Strategies Using Logistic Growth Model, 2012.

[6] Corinne Wentworth, Optimum harvesting models for fishery population, summer of 2011.

[7] T. K. Kar, and Bapan Ghosh, Impacts of maximum sustainable yield policy to prey-predator systems, 2013.

[8] Predation, Wikipedia, the free encyclopedia.

[9] K. Madhusudhan Reddy and K. Lakshmi Narayan A PreyPredator Model with an Alternative Food for the Predator and Optimal Harvesting of the Prey, 2011.

[10] Tapan Kumar Kar and Kunal Chakraborty effort dynamics in a prey-predator model with harvesting, International journal of informatics and system science 2010.

[11] A brief explanation of biomass and maximum sustainable yield, Ministry of fisheries, July 2006. 